\title{
Theoretical aspects of problem solution in production systems
}

\author{
Vitaly Sergeevich Borovik ${ }^{1, *}$, Dmitry Anatolevich Skorobogatchenko ${ }^{2}$, Vitaly Vitalievich Borovik ${ }^{3}$, Oksana \\ Vladimirovna Procenko ${ }^{3}$ and Anastasiya Vitalievna Borovik ${ }^{3}$ \\ ${ }^{1}$ Volgograd Science and Technology Center, Komsomolskay 12, Volgograd, Russia \\ ${ }^{2}$ Volgograd State Technical University, Akademitheskay, 1, Volgograd, Russia \\ 3 Department of Capital Construction of the State Public Institution of Volgograd Region \\ «Directorate of Highways», Zukova 62, Volgograd, Russia.
}

\begin{abstract}
The article attempts to present conceptually the definitions of the terms a "problem situation" and a "problem". The problem, goal, strategy, means and conditions are analyzed in time, in interconnection and interdependence. A scheme for the solution of a problem based on the digital strategy model is presented. The one-to-one correspondence of the elements of the sets within the implementation of the corresponding measures connected with the solution of the problem is analyzed as an illustration of the way out of the problem situation. The model is analyzed which shows the time sections of the Minkowski space for the control vector, taking into account the reaction of the control vector to the influence of external and internal factors in time within the production system. The conclusion is made about the evolutionary reaction of the system to the emergence of a problem situation, which is formed for its self-preservation, prevention of system integrity violation and the possibility of automating the process of problem solution on the basis of a digital model.
\end{abstract}

\section{Introduction}

Joseph Schumpeter in the introduction to his work [1] writes: "This is because, whatever the field, the problems and methods that are in use at any given time embody the achievements and carry the scars of work that has been done in the past under entirely different conditions. The significance and validity of both problems and methods cannot be fully grasped without knowledge of previous problems and methods to which they are the (tentative) response". An important prerequisite for the successful solution of problems is their adequate definition in time.

Taking into account all the variety of information reflecting the study, research and resolution of various problems, as a rule, there are no clear indications of what, in fact, the authors see the problem, for example [2-8]. Hence, it can be assumed that at present time, intuition plays a leading role in understanding the essence and content of what can or should be seen as a problem. However, intuitive understanding does not contribute to emphasis of the main thing, i.e. a controlled result.

It is very difficult to concentrate resources accurately enough, to balance their consumption in the most critical areas connected with the problem solution. Unclear wordings disorient the workers.
Unclear understanding prevents the identification of the main direction of work, balance in the use of resources, justification and critical analysis of problem solution. This situation affects the way in which the activities are coordinated, leading to a significant waste of resources. As J. Schumpeter writes “ ... the highest claim that can be made for the history of any science or of science in general is that it teaches us much about the ways of the human mind. To be sure, the material it presents bears only upon a particular kind of intellectual activity. But within this field its evidence is almost ideally complete. It displays logic in the concrete, logic in action, logic wedded to vision and to purpose" [1]. The absence of a clearly defined problem does not contribute to an unambiguous formulation of the goal and strategy for its achievement. According to Aristotle: "As the most important and dominant science, which all other sciences, like slaves, do not dare to contradict, should be called wisdom of the science of the purpose and the good (as for their sake there is something else)" [9].

The concepts that have developed to date on this topical issue do not fully meet the requirements of consistency. The aim of the work is to study the problem, goal,

*Corresponding author: borovikv@mail.ru 
strategy, means and conditions in time, interconnection and interdependence.

\section{Research methodology}

Aristotle suggested the division of problems into three groups: practical, theoretical and those which serve to achieve any truth [10]. In practice, situations constantly arise, the resolution of which with the help of available means, knowledge and experience is complicated [11]. In such cases, it is customary to say that there is a problem. The problem, like the task, comes from a problem situation $[12,13]$. The appearance of a problem situation is connected with the emergence of information (Fig. 1) [14,15].

The analysis of a problem situation creates the prerequisites for its transformation either into a task or into a problem. For our further research, we will consider such a division of the problem situation sufficient for all the variety of interpretations of the problem concept $[16,17]$.

In our opinion, in production systems, the problem situation manifests itself in information about the discrepancy between the actual result and the desired one, or real conditions and desired means. Inconsistency in such systems arises under the influence of information, which, as a rule, has the status of a document.

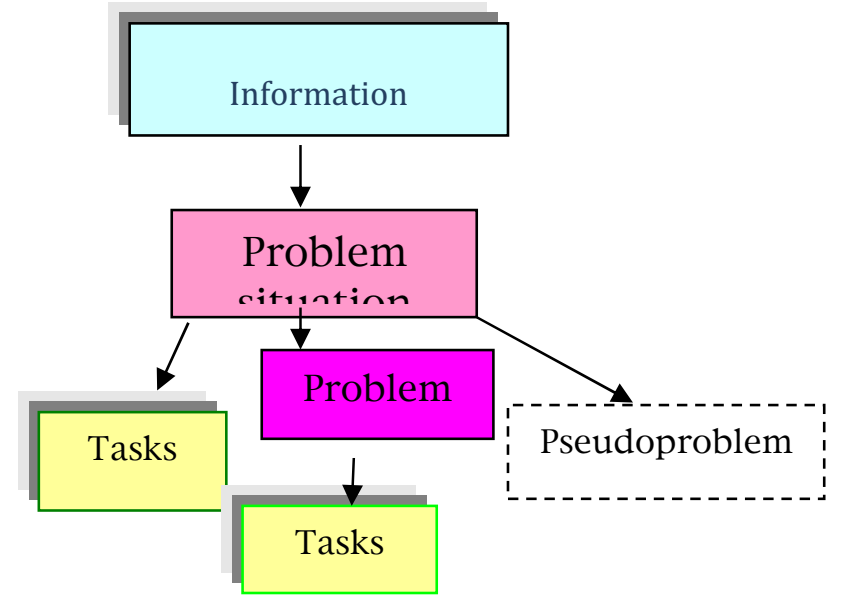

Fig.1. Structural diagram of the process of problem situation transformation

The types of problems, depending on the field of activity, can be scientific, industrial, social, political, etc. [17]. In terms of the scale of influence, production problems can be local, regional, sectoral, state, global, etc. Each type and scale of influence of a problem situation requires its own individual approach to its solution.

For our study, the problems associated with the production activities of enterprises, for example, road construction industry, are of interest. As a rule, an inaccurate definition of the goal, areas, scale of influence, errors in the process analysis of the conditions, means and time in which a problem situation arose, leads to a pseudo-problem [19]. The methodology and techniques for problem solution isolated from problem situations have been developed in sufficient detail, for example in the paper [20].

The process of recognition, isolation and solution of production problems has not been sufficiently developed. The available recommendations present the descriptive part of the problem in sufficient detail, without defining it, for example in the paper [21]. In our opinion, a problem situation manifests itself in the course of comparing information about the results of production process at the current moment with information about the results that had been planned earlier. The discrepancy between the values of the compared parameters and the absence of a solution at a given moment of time causes a problem situation if the parameters of this discrepancy go beyond the limits established earlier.

For example, in accordance with the contract between an owner and a road construction company, a road was built with a five-year warranty. Three years later, it was documented that in terms of its transport and operational state the road does not meet regulatory requirements. The warranty was not honored. It was established that the transport and operational state of the road does not correspond to the planned transport and operational state and, in terms of its parameters, goes beyond the previously established limits. A problem situation arose.

The occurrence of the problem is associated with information about the discrepancy between the results of 
production activities at the current moment and the results that are planned for a long term period, also if this discrepancy goes beyond the established limits. In this situation, the time becomes a sign of the distinciton between a problem situation and a problem.

For example, the management of a road construction company may have two main ways out from the problem situation mentioned above.

1. To solve the problem, i.e. develop a repair project for the restoration of the transport and operational state of the highway and carry out a set of works in accordance with the project. It takes time $t$ and resources to get out of a problem situation $\sum_{i=1}^{n} X_{i}$.

2. To refuse to honor the warranty obligations, which will create the preconditions for a problem, because the customer can submit the relevant documents to the arbitration court and he is likely to win the case with subsequent sanctions. In addition, for the road construction company in the future, it may also be difficult to conclude contracts, etc. It will take time $t_{1}$ and resources to solve the problem $\sum_{i=1}^{n} X_{i}^{\prime}$. At the same time, as a rule, $t<t_{1}$ and $\sum_{i=1}^{n} X_{i}<\sum_{i=1}^{n} X_{i}^{\prime}$.

The concept what we suggest is consistent with the position of the author [22], who, emphasizing the method for problem identificaiton, notes that it is based on the "assessment of the state of a controlled system founded on the results of scenarios of self-development and controlled development." In the authors' opinion, the "scenario results" can be seen as information about possible predicted events, i.e. planned for a future period. Thus, a common feature of a problem and a problem situation is information about the inconsistency of the results. The difference between a problem situation and a problem lies in the fact that the occurrence of a problem situation is connected with the appearance of information about the discrepancy between the planned results and the results available in the current period of time, and in case of a problem it is about the results of the current period and the forecasted one. It should be emphasized that the emergence of a problem situation is strictly connected with the goal, strategy, conditions, means and time. A change in goal and strategy can lead either to a task or to a problem if the problem situation is ignored.

\section{Research results}

A strategy for problem solution is understood as a plan of actions coordinated by enlarged indicators in space and time, which in a certain way ensures the achievement of the main goal on the basis of adequately used conditions and means.

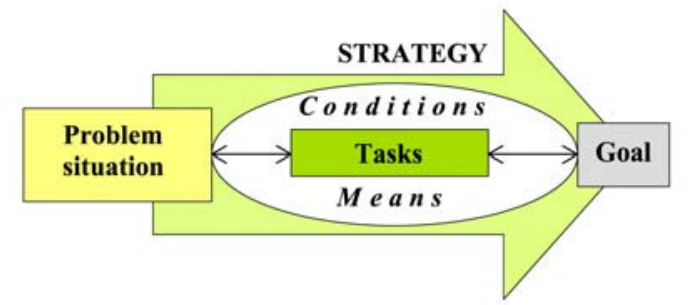

Fig 2. Diagram for a problem solution based on a strategy

The need to solve the problem is associated with the emergence of a problem situation. Objectively, the emergence of a problem situation is connected with the process of production development and progress.

In order to get out of the problem situation, it is necessary that each finite set of available factors $A$ (conditions, means) corresponds to a finite set of necessary factors $B$, which ensure the solution of the problem (Fig. 2). Then we can write

If the sets $n$ do not intersect each other, i.e.

$$
A_{n} \bigcap A_{n^{\perp}}=\varnothing \text { then } n \neq n^{\prime}
$$

and the sets $n$ also do not intersect each other, i.e.

$$
B_{n} \cap B_{n^{3}}=\varnothing \text { at } n \neq n^{\prime} .
$$

For all $n A n \sim B n$. Then each

$$
a_{0} \in \bigcup_{n=1}^{m} A_{n},
$$

matches a single element 


$$
b_{0} \in \bigcup_{n=1}^{m} B_{n} .
$$

The correlation of each element of the set $A$ according to some rule to one and only one element of the set $B$ and when correlating each element of the set $B$ to one and only one element from the set $A$, then

$$
\bigcup_{n=1}^{m} A_{n} \sim \bigcup_{n=1}^{m} B_{n} .
$$

One-to-one correspondence (6) in the implementation of appropriate measures connected with the solution of the problem can be considered as evidence of the way out of the problem situation. Information about the problem situation gives an impetus for collecting, systematizing and analyzing information that allows us solving a problem or defining the problem. For further analysis, it is important to note that the problem situation after the division into tasks and problems may arise again when solving a task or a problem. The emergence of problem situations, problem solution and forecasting problems is a constantly evolving process.

As a rule, such situations are aggravated by the innovative development of production, in conditions of a shortage, primarily of time, as well as information about new technologies, materials, and processes. The desire to find a particular time in the development of the processes occurring in the production system in terms of advanced technology, leads to a number of assumptions that deserve serious attention $[24,25,26]$.

J. Schumpeter pointed out the possibility of modeling production processes in time, mentioning the introduction of a time parameter into the production function: "If we want to base our theory of production on the theory of the Jevons-Böhm-Bawerk-Taussig "roundabout" process, we can introduce the notion of time directly into the production function by writing $x=$ $\psi\left(v_{1}, v_{2} \ldots v_{\mathrm{n}} ; t\right) \ldots \gg[1]$.

Let us turn to the production function (we consider it as a digital model), for example, in the following form [28]:

$$
Y=C_{0} \prod_{i=1}^{n} X_{i}^{\alpha_{i}}
$$

where $Y$ is the calculated index (for example, the amount of work, etc. in physical-material or value terms); $i, i=\overline{1, n}$ - factors (for example, fixed assets, materials, labor, energy and qualifications of personnel) that determine the parameters $Y$ (in physical-material or value terms); i, $\mathrm{i}=\overline{\mathrm{i}, \mathrm{n}}$ characterize the contribution of $i$ into $Y ; C_{0}$ is a coefficient reflecting the influence of factors which were not taken into account by the model.

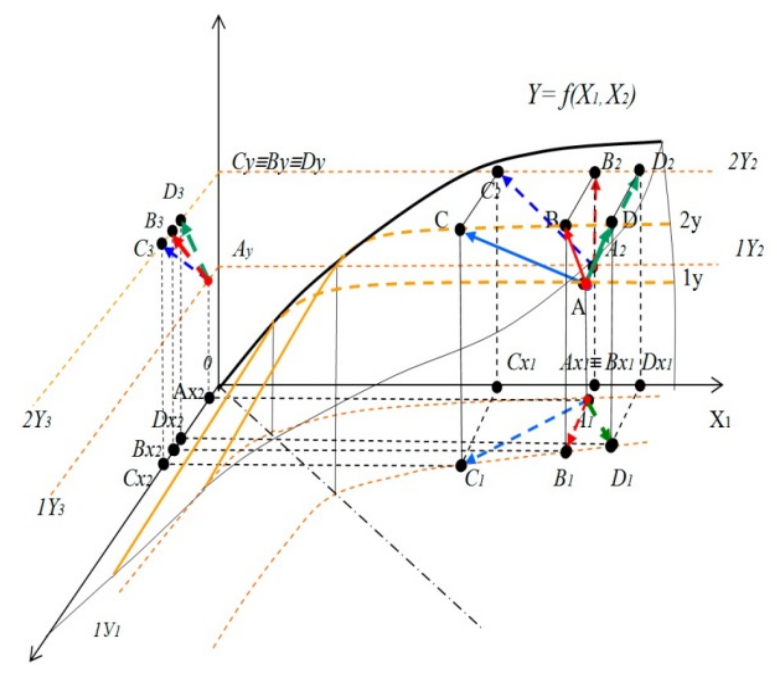

Fig. 3. Three-dimensional model based on production function, displaying a surface illustrating control options when solving the problem of output from $1 y$ to $2 y$.

For example, a digital model of the type $Y=1.132 \cdot X_{1}^{0.763} \cdot X_{2}^{0.438} \cdot X_{3}^{0.211} \cdot X_{4}^{0.176} \cdot X_{5}^{0.076}$ was obtained and it characterizes the hypersurface of the produciton process under study. Using the obtained digital model, it is not difficult to determine the mathematical model of the control strategy. For graphic interpretation of a digital model in three-dimensional space, it is advisable to choose the most influenced factors, for example, $X_{1}$ and $X_{2}$. (Fig. 3), [28]. Curves $1 Y$ and $2 Y$ connect points with the same numerical values of $Y$. Their projections $1 Y_{1}, 2 Y_{1}, 1 Y_{2}, 2 Y_{2}, 1 Y_{3}, 2 Y_{3}$ are isoquants.

In Figure 1, point $A$, has a numerical characteristic, for example, the amount of work $1 Y$. The $2 Y$ the scope of work can be achieved by introducing, for example, a new technology, an infinite number of combinations of $X_{1}$ and $X_{2}$. For example, let'is analyze points $B, C$ and $D$, illustrating three options for the achievement of the amount of work, characterized as $2 Y$. Depending on the purpose, conditions and means, one or another variant of the combination of the use of resources is selected. From the mathematical point of view, the optimal strategy for reaching $2 Y$ should be recognized as the vector $\mathbf{A B}$, since $\mathbf{A} \mathbf{B} \perp 2 y$ and it is the shortest distance between the two curves $y_{1}$ and $y_{2}$, as a perpendicular to the tangent at point $B$.

It should be noted that the use of a digital model makes it possible to unambiguously determine the optimal control strategy.

Let us exemplify the emergence of a problem situation by the development of production function in time. Let us suppose that the beginning of some 
production process is fixed at point $A$. Time is associated with the consumption of resources and their movement in space, connected with the production process. The "past" of the process coincides with its beginning, and time "flows" along with the process of resource transfomraiton in space [28].

Hence the conclusion is that time conditionally flows in the same direction as the changes of the observed object. Motion, as a generalized concept, is usually characterized by vector quantities and it can be assumed that the "proper" time of the process will be collinear and co-directional with the "displacement" vector. Let us exemplify this graphically using the geometric interpretation of space and time (Fig. 4) [18, 28 - 31].

In real production conditions, control in space and time begins from point $A$ (from the achieved result) in the direction of point $B$ (the planned result, for example, production of a good). The actual geometric development of the process occurs over time $A^{\prime}$ from to $B^{\prime}$. It is worth mentioning that this process corresponds to a certain time or the planned time of commodity production. Depending on the processes associated with the control developing in time point $B$ of the vector $\mathbf{A B}$ it describes a rather complex trajectory (Fig. 4).

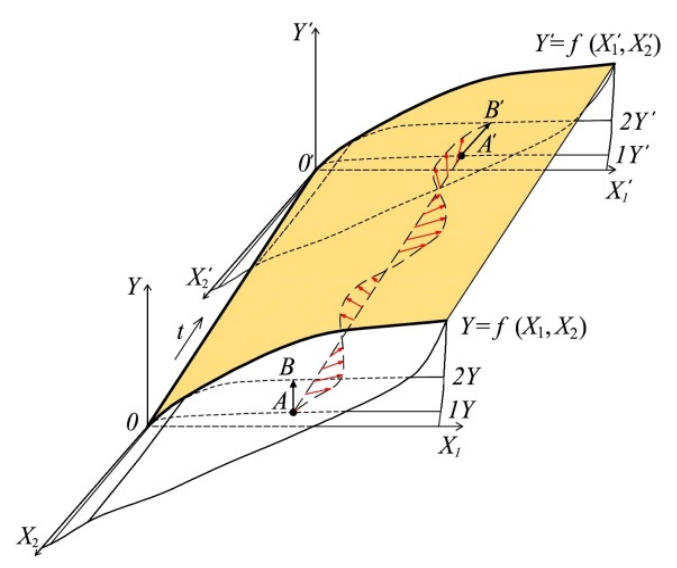

Fig. 4. A model exmplifying the time sections of the Minkowski space for the control vector $\mathbf{A B}$ (transformations $\mathbf{A B}$ into $\mathbf{A}^{\prime} \mathbf{B}^{\prime}$ ), taking into account the reaction of the control vector to the influence of external and internal factors in time in the production system

It is explained by the fact that the system of mobile equilibrium tends to change in such a way as to minimize the effect of external influences [32]. The influence of a set of factors developing in time, i.e. changing conditions, changing means, delays in the reaction of the control system to changes in the production process, including overregulation [33], and other reasons, the trajectory of the movement of the control vector in time in a general form can be presented for example, in the form of a wavy surface (Fig. 4).

As it can be seen from Figure 4, the position of the vector $\mathbf{A}^{\prime} \mathbf{B}$ ' is not the shortest distance between $1 Y$ and $2 Y$. and it indicates a problematic situation.

\section{Discussion of the results}

In a problem situation, it is necessary to clearly identify either the problem or the task. To identify this means, first of all, to define accurately the task or problem. Most clearly, this can be done using the the strategy as the main goal of the enterprise or an organization. The emergence of a problem situation is associated with a goal.

If, in order to achieve the goal set previously at a specific point in time, the discrepancy of conditions or means is found out, then this indicates the occurrence of a problem situation. Innovative development involves setting goals that determine a more intense way of functioning of the organizational and economic system. As a rule, innovations are based on a more efficient use of available funds (resources, including time) [34] and production conditions. Therefore, we can state with confidence that innovative development creates the preconditions for the emergence of problem situations and objectively contributes to the production.

It is extremely important for practice to determine the signs and properties of a problem situation. It is necessary to identify the basic requirements that play an important role in understanding and definintion of problems, as well as effective methods for their solutions. A detailed study of these issues allows us separating at an early stage the real problem from the pseudo problem.

A problem situation is transformed into a task if a way out of this situation is found with the help of information about the available means and conditions for problem solution (Fig. 1, 3). In contrast to the task, the problem is perceived as a contradictory situation. It is characterized by the need to perform certain actions, for which at a particular moment in time there is no necessary information about possible options for the transformation of funds or conditions for its solution. The emergence of production problems as a rule is associated with the lack of information about the possibility of getting out of the problem situation by means of the solution of the problem. To obtain such 
information, as a rule, it is necessary to perform a set of special actions, for example, research and development, etc. [35].

For example, a problematic situation can be connected with the supply of stone materials to facilities under construction, which are significantly far from railway communications. You can get out of this problematic situation by solving the problem, i.e. using road transport.

If we stop using road transport as a very expensive method of delivering materials, then the problematic situation will be transformed into a problem. One of the ways to solve the problem could be, for example, the use of local soils as the base of the pavement. However, there is no information on the appropriate effective technology that meets the regulatory requirements at a specific point in time for specific conditions in the enterprise. To solve this problem, it is necessary to conduct research, tests, production experiments, develop technology, regulatory documents, aggre documents, approve papers, purchase the necessary equipment, etc. A combined option is possible, to conduct a complex of research and development work simultaneously with the solution of the problem, i.e. using vehicles to solve the problem.

Taking into account the desire of any system of mobile balance to minimize the effect of external influence, evolutionary protection in the form of self-preservation has been formed in the production system. However, the influence of unresolved internal and external problems can lead the system to an unsatisfactory state, to a violation of the integrity of the system.

The integrity of the system is a fundamental nonreducibility of the properties of the system to the sum of the properties of its constituent elements and the nondeduction from the last properties of the whole; dependence of each element, its properties and relations in the system on its place, functions, etc. within the whole [36].

In dynamics, this means that the impact on one element of the system (or some of them) necessarily causes a reaction, a change in other elements (dynamic integrity). For example, if the plans of an enterprise are integrated into a system, then a change in demand for products will entail adjusting the tasks for supply, for labor, perhaps for capital investments, cost and other indicators [37].

However, the violation of the integrity of the system can also be expressed by the transition of the system to a new qualitative state. In the system, under the influence of resources coming from outside, there is a slow quantitative accumulation of insignificant changes. This occurs up to a certain limit, beyond which a radical change in its state is observed, which can be carried out almost instantly, in a jump.

The system temporarily finds itself in an unstable state, "loses its memory," and the nature of its subsequent development is determined only by those random factors that act on the system at that moment. In order to get out of it, the system has the following opportunities: degradation, destruction, involution or self-organization, complication, evolution. Quantitative changes turn into structural or qualitative ones, and the whole process of system development can be presented as a series of slow and abrupt changes replacing each other. Each of these changes can be presented as a transition of the system to a new qualitative state [38]. This fact reveals a contradiction. On the one hand, there is a desire for self-preservation, and on the other, there is the need for improvement.

\section{Conclusion}

An attempt is made to conceptualize the content of the definitions of problem situation and problem. As a starting point, a significant difference between the concepts of task, problem situation and problem is accepted. It is shown that the problem, goal, strategy, means and conditions should be analyzed in time, in interconnection and interdependence. The possibility of problem solution based on a digital strategy is shown. A one-to-one correspondence of the elements of the sets was established during the implementation of appropriate measures connected with the solution of the problem, as evidence of the way out of the problem situation. A 
model was developed that exemplified the time sections of the Minkowski space for the control vector, taking into account its response to the influence of external and internal factors in time in the production system. It is found that the evolutionarily system response to the occurrence of the problem situation is created for preservation, prevention of disruption of the integrity of the system. The manifestation of the contradiction in the trend of the system for selfpreservation on the one hand, and the need for its improvement on the other is shown. The digitization of the process of getting out of a problem situation creates the prerequisites for its automation.

\section{References}

1. J. A. Schumpeter History of Economic Analysis. (London: Taylor \& Francis eLibrary, 2006)

2. Proceedings of the international scientific and technical conference. (Bryansk State Engineering and Technological Academy. Bryansk. 1998).

3. Problems of technology improvement of material resources saving. Interuniversity collection of scientific papers of the Kazakh Polytechnic Institute named after V.I. Lenin. (Alma-Ata, 1987).

4. Problems of improvement of planning and forecasting of capital construction. Interuniversity thematic collection of works of the Leningrad Civil Engineering Institute. (L .: 1981).

5. E.E. Rumyantseva New Economic Encyclopedia. (Moscow: Infra-M. 2006).

6. New Economic Dictionary. (Moscow: Institute of New Economy. 2006)

7. V. Lukyanova Problems of technical regulation reform. Standard and quality 12 (2006)

8. Problems of the construction complex of Russia. Materials of the 10th interhnational scientific and technical conference at the 10th international specialsized Exhibition "Construction and utilities - 2006" (Ufa, 2006).

9. Aristotle. Works in four volumes. Volume. 1. (Publishing house of socio-economic literature "Mysl", 1976)

10. Aristotle. Works in four volumes. Volume 2. (Publishing house of socio-economic literature "Mysl". 1976)

11. I.G. Gerasimov Research structure. (Moscow, "Mysl", 1985).
12. A Brief Psychological Dictionary. (Moscow, Publishing house of political literature, 1985).

13. Philosophical Encyclopedic Dictionary. (Moscow, Soviet encyclopedia, 1983)

14. D.A. Molodtsov Stability of the principles of optimality. (Moscow: Science. 1987).

15. Logical dictionary-reference. (Moscow, Science. 1975)

16. Types and levels of problem formulation. Retrieved from: http://www.trizminsk.org/e/248009.htm.

17. V S Borovik Transportation Research Procedia. 20, 74-79, (2017)

18. P.A. Vasev, Proceedings of the 12th International Conference on Computer Graphics and Machine Vision GrafiKon. P. 431-437 (Novgorod, 2002).

19. I.G. Gerasimov Research structure. (Moscow: Mysl., 1985).

20. A.G. Teslinov Development of control systems: methodology and conceptual structures. (Moscow: "Globus". 1998)

21. F. Heiligen Formulation of the problem of posing problems in: Cybernetics and systems ' 88 , Available http://pespmc1.vub.ac.be/Papers/ProblemFormulation.html.

22. Z. K. Avdeeva Methods for the formation of strategies for solution of semi-structured problems based on cognitive models. (Abstract of the thesis for the degree of technical sciences) Institute of Control Sciences of the Russian Academy of Sciences. (Moscow, 2006).

23. R. R. Stoll 1967 Sets, Logic and Axiomatic Theories. (W.H. Freeman and Company. SanFrancisco and London, 1967).

24. V.P. Kazaryan Concept of time in the structure of scientific knowledge. (Moscow: Publishing house of Moscow State University, 1980).

25. Smolin Lee, The trouble with physics: the rise of string theory, the fall of a science, and what comes next. (Houghton Mifflin, Boston, 20016).

26. M.T. Zinaliev Physics of Time (LAP LAMBERT Academic Publishing, 2015)

27. G.B. Kleiner Production functions. Theory, methods, application. (Moscow: Finance and Statistics, 1986)

28. V.S. Borovik, V.V. Borovik, Eastern-European Journal Enterprise Technologies 3/5 (81), 4-10, 2016. Doi:10.15587/1729-4061.2016.69383

29. H. Minkowski, Geometrie der Zahlen. (LeipzigBerlin: R. G. Teubner, 1910)

30. A. Einstein The essence of the theory of relativity. (Foreign Literature Publishing House, 1955).

31. A.B. Fashchevsky Graphical representation of four-dimensional space. Retrieved from: http://khd2.narod.ru/authors/fshvsky/spc4d.htm.

32. A.A. Bogdanov Tecktology: A General Organizational Science. (Moscow, 1989)

33. V.S. Borovik, A.V. Borovik. IOP Conference Series: Materials of Science and Technology, 832, $012044 \quad$ (2020) doi:10.1088/1757899X/832/1/012044. 
34. V.S, Borovik, V.V.Borovik, Eastern- European Journal of Enterprise Technologies. 6, 10(84), 41-48( 2016) DOI: 10.15587/17294061.2016.86535.

35. A. Kondakov From the materials of the "Intellectual Club". (St. Petersburg, 1997).

36. System integrity. Retrieved from: https://economic_mathematics.academic.ru/4939/

37. L.I. Lopatnikov Economic and mathematical dictionary: Dictionary of modern economic science. 5th ed. (Moscow: Delo, 2003).

38. Synergetics as a universal scientific paradigm. Retrieved from: http://knowledge.allbest.ru/biology/2c0b65625b3 ac68b5d53b88421306d27_0.html. 